


\section{Goethes fauft}

erflärt von

\section{adolf Trendelenburg}

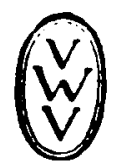

Serlin uno Leipzig 1922

Dereinigung wiffenfdaftlicter Derleger walter be Gruyter \& Co.

vorm. B. I. Böfden'fḑe Derlagshandlung - I. Buttentag, Derlagsbuфhạdilung - Beorg Reimer - Karl I. Cräbner Deit \& Comp. 


\title{
Goetbes faupt
}

erflärt von

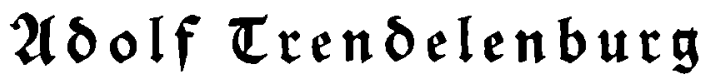

\author{
Der Eragöbie erfter Ceil
}

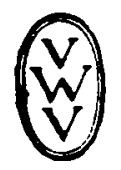

Berlin uno Reipsig 1922

Dereinigung wiffenfthaftid)er Derleger walter be Gruyter \& $C_{0}$.

vorm. B. J. Bölden'fhe Derlagshandlung - I. Buttentag, Derlagsbadbandlung - Georg Reimer - Karl J. Crübner Deit \& Gomp. 
Drud ber Dereinigung miffenfhaftliaer Derleger wolier be Bruỵter \& Co., Berlin W. 10. 


\section{3um Geleit}

it der Ģauptoerjammlung der Boethe=Befelljhaft $\mathfrak{j}^{\text {u }}$ Weimar, den 21. Mai 1921, erhielt der Derfafier das Wort $\mathfrak{\jmath}^{\mathfrak{u}}$ folgenden Jusführungen:

"Ih bitte um die Ehre, die beiden erften und vor= läufig einjigen Exenularc meines fauftFomnentars der Goethe $=$ Bibliothel und dent Goethe $=$ und Saillerardive iiberweifen und jugleid mit menigen Säzen das Wagnis

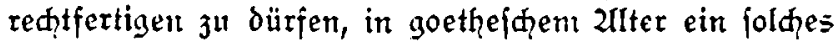
Werf ju unternehmen.

So treffliạ die crrift ju nehmenden Sdiriften find, die fid der fauptertärung wiomen, fo haften bod allen gemiffe Mängel an, die fid ungezwungen aus bem Stu= diengange ihrer fajt ausjdilteblid deu afademijąen Kreifen der Bermanifter und siterarhiftorifer angehörenden $\mathfrak{D e r}$ faffer erflären. Mit denl Flaffificien Xltertum, in dem Boethe mit allen fajern wurjelt, find fie, fozujagen, nur im Zebenamte verbunden; mit fragen der bildenden Kunft, der Goethe die zahlreiditen und tiefiten Źnregun= gen verdant, haben nur wentige fid eingehender ber

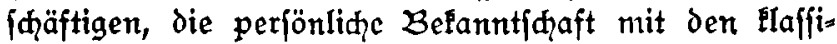

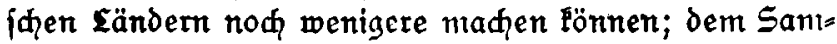
meln päbagogifąer Erfahrungen endią erweift fid ałademiface Eehrtätigleit niat immer förderlich. Daher fommt bei den bisherigen Kommentaren die auffallende

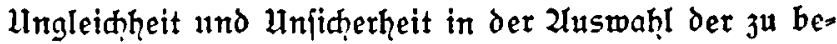


fpreatenden Dinge. Sie fpringen von cimem zun andern, taften hiter und dorthin, ergehen fid weilläufig an Stellen, die der Erflärung befondere Sdịierigleiten nid̨t bieten, und übergehen dafür wirflid dunlle; genug, fie verfahren willfürrlia, wo ftrenge pädagogifţe Grundjätze am Plaţc mären.

Berade an diejen punte aber glaube id mid be= rufen, $3^{u}$ ḩelfen und $\mathfrak{j}^{\prime}$ beffern. In meiner halbhundert= jährigen Cätigleit als sehrer und Eciter humaniftifher Bymnajien habe id reide Belegenḩeit gehabt, päba= gogifhe Erfahrungen ju maçen und die Bedürfniffe derer Fennenzulemen, die in das Derftänonis fapwieriger Shriftiteller eingeführt werden follen. Don der llafí \{ąen philologic jur Urąäologic unt von diejer jur Kunit= gefchid)te geführt, bin ia erit auf dicfem weiten, boch. nidht unfmatbaren Unwwege $\mathfrak{j}$ Boethe und feinem faupt gefommen. Zud ift mir das Glï gemorden, brei volle Jahre in Jtalien unter andcrem ouț auf Goethes Spuren jิu wandeln.

So ausgeriiftet madite id mid, wit in der Unraft des

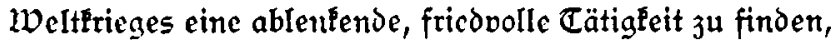
an die Zziederiantift des Konmmentars, wobei mir mein Ulter niait wur als Fein Gindernis, fondern gerabezu als Worzug eridien, wenn id art Mephiftos Worte dahte, die er dem jüngeren, niạt applaudierenden parterre зumft:

Jḩr bleibt bei meinen worte Falt, Eud guten Kindern Iaff' id's gehen;

Bedentt: der Ceufel, der ift alt, So werbet alt, ihn zu verftehenen.

Ia begann meine Yusarbeitung mit dem 3 weiten Teile des Dramas, weil gerade er dent Flafififten philo.

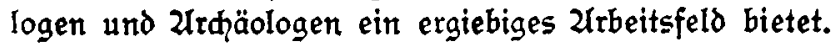


Dant der Energie und Seiftungsfähigleit des Derlages ift der niat eben einfahe Drud, obwohl erft im februar begonnen, dođ, bis zuin Pfingftfeft fo weit gefördert

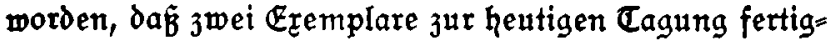
geftellt werden fonnten. Wenn das $\mathcal{B}_{\text {uq }}$ heute fo viel Foftet wie vor dem Kriege eine ganze Klaffiferbibliothel, fo if das \{amerzlia, aber leider niđht zu änderm. Eröjt= Iiđ wenigftens bleibt dabei, daß̧ in Drud, papier und Uusftattung etmas friedensmähiges erreid̨t morden ift und das Bud jeinem Befiger wohl eine Ileine fauftbibliothe crietzen Fann.

Ia pläbiere vor diefer maß̧gebenden Deriammlung für mein Wagnis niąt auf mildernde $l$ lmf́tände. Im Begenteil, id werde and frenge Urteile und Belehrun= gen gern und danfbar anneb̧men; dent ia bin der luber jeugung, daḱ nur ernjtes $\mathfrak{Z} u$ jammenarbeiten vieler die

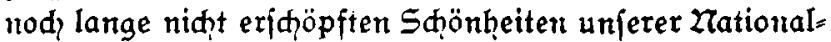
diḑtung nad und nad uns enthüllen wird. Unierer zrationaldiatung! wahrlia, es tut bejonders in den wirren ber Begenwart bitter not, baß wir Deutjąe uns auf unjere Zationalität befinnen. Dazu aber Fönnen wir einen befferen führer nidit wählen als das alte Weimar und feinen Boethe."

Im Geleitwort des Pfingiten 1921 erjhienenen Bandes ift das Eriaheinen des Kommentars $\mathfrak{u}^{\mathfrak{u} m}$ erften Teile des faujt "etwa in Jahresfrift" in Uusfidit geftellt. Dies Deripredien hat jia ciulöjen Iaffen. Die bewäh̆ten Riatlinien find aud hier feftgehalten worden. Da bem eriten Ceile des Dramas cine Crennung in Ufte fehlt, mugte die gefamte Einleitung dem Kommentar voran= geffellt werden. Sie enthält die gleiden Ubjanitte wie die Sondereinleitungen $\hat{\jmath}^{\mathfrak{u}}$ den fünf $\mathfrak{X}_{\text {flten }}$ des 3 meiten Ceiles Rrbeit des Didhters, Perjonenüberjiąt, Đrtlia Eeiten, Bang der Gandlung, Einzelheiten). Die Jer 
legung in einen fauft und einen Gretđhenteil, die Jählung

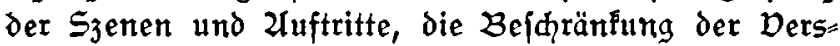
jäh̆lung auf $S_{j}$ enen und $S_{j}$ enengruppen ift Iediglid der

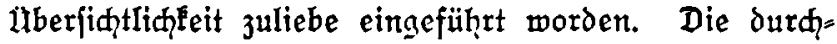
gehende Derszählung der weimarer Zusgabe findet maan als überiarift jeder rechten Seite.

Das Problem des Goetheinen fault, hat einmal Guftav Roethe gejagt, liegt im crfien Ceile. Die Wah̆ = heit diejes wortes hat fit dem Derfaffer bei der 2lus: arbeitung in erftaunliḑent Hunfange beftätigt. Weite Streden des vorliegenden Bandes Iegen davon Jeugnis ab. Es zeugt das ebenjojeḩr fiur den unerjä̈ppliđen Gedankenrei⿱千口tam des Dramas mie für das unjulängliđg̣c Vermögen jedes einzelnen Erflärcrs. Der Derfaffer ift Feinem ernfthaften Problent aus dem Wege gegangen.

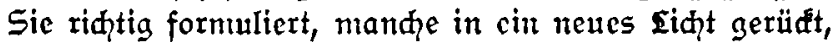
cins und das anderc vielleidft cincr Eöfung näher sefühţrt $\mathfrak{\jmath} \mathfrak{u}$ ḩaben, ijt feine Goffnung.

Berlin, Pfingften 1922 